\title{
Association between Dehydroepiandrosterone-sulfate and Attention in Long-term Survivors of Childhood Acute Lymphoblastic Leukemia Treated with Only Chemotherapy
}

\author{
Yin Ting Cheung ${ }^{1}$, Wassim Chemaitilly ${ }^{2}$, Daniel A. Mulrooney ${ }^{1,3}$, Tara M. Brinkman ${ }^{1,4}$, Wei \\ Liu $^{5}$, Pia Banerjee ${ }^{1}$, Deokumar Srivastava ${ }^{5}$, Ching-Hon Pui ${ }^{3}$, Leslie L. Robison ${ }^{1}$, Melissa M. \\ Hudson ${ }^{1,3}$, and Kevin R. Krull ${ }^{1,4}{ }^{*}$ \\ ${ }^{1}$ Departments of Epidemiology and Cancer Control, St Jude Children's Research Hospital, \\ Memphis, USA \\ 2 Departments of Endocrinology, St Jude Children's Research Hospital, Memphis, USA \\ 3 Departments of Oncology, St Jude Children's Research Hospital, Memphis, USA \\ ${ }^{4}$ Departments of Psychology, St Jude Children's Research Hospital, Memphis, USA \\ ${ }^{5}$ Departments of Biostatistics, St Jude Children's Research Hospital, Memphis, USA
}

\section{Abstract}

Long-term survivors of childhood acute lymphoblastic leukemia (ALL) are at risk for neurocognitive impairment and may be at risk for compromised hypothalamic-pituitary-adrenal (HPA) function. Dehydroepiandrosterone-sulfate (DHEAS) is an adrenal androgen commonly used as a marker of HPA function. In the general population, a low level of DHEAS has been associated with poorer cognition. At $\geq 2$ years post-treatment, we examined the association of DHEAS with attention outcomes in 35 male and 34 female long-term survivors of childhood ALL (mean[standard deviation] age at evaluation 14.5[4.7] years; 7.5[1.9] years post-diagnosis) who were treated with only chemotherapy and without prophylactic cranial irradiation. Male survivors with low-normal levels of DHEAS had worse performance than male survivors with high levels of DHEAS on multiple measures of attention (all $P$ s $<0.05$ ). However, association between DHEAS and attention measures were not found in female survivors. Our results suggest that survivors of ALL who suffer from partial but persistent adrenal insufficiency may be at risk for neurocognitive deficits. This finding should be validated in a larger prospective study, with attention to sex differences in the potential impact of adrenal insufficiency on neurocognitive outcomes.

Corresponding Author: Kevin R. Krull, PhD, Department of Epidemiology and Cancer Control, St. Jude Children's Research Hospital, 262 Danny Thomas Place, MS 735, Memphis, TN 38105-3678; (v) 901-595-5891; (f) 901-595-5845; kevin.krull@ stjude.org.

Publisher's Disclaimer: This is a PDF file of an unedited manuscript that has been accepted for publication. As a service to our customers we are providing this early version of the manuscript. The manuscript will undergo copyediting, typesetting, and review of the resulting proof before it is published in its final citable form. Please note that during the production process errors may be discovered which could affect the content, and all legal disclaimers that apply to the journal pertain. 


\section{Keywords}

Attention; Chemotherapy; Childhood acute lymphoblastic leukemia; Dehydroepiandrosterone sulphate; Neurocognitive function

\section{Introduction}

Acute lymphoblastic leukemia (ALL) is the most common childhood malignancy. Despite the gradual omission of prophylactic central nervous system irradiation as a treatment modality (Pui et al., 2009), survivors of childhood ALL may still experience treatmentrelated late effects due to the exposure to multi-agent intravenous and intrathecal chemotherapy. Our research team has found that at more than 5 years post-diagnosis, longterm survivors of ALL who were treated with a contemporary chemotherapy protocol without cranial radiation demonstrated rates of attention problems higher than age-based normative data (Krull et al., 2016). Current research is now focused on the identification of the source and reduction of factors that contribute to these persistent neurocognitive deficits.

Survivors of childhood ALL are also at risk for endocrine disturbances, including precocious or delayed puberty, thyroid dysfunction, and metabolic disorders. Although these endocrine complications are predominantly associated with radiation, a recent report of 14,290 survivors from the Childhood Cancer Survivor Study indicated that, at 5 or more years postdiagnosis, survivors were significantly more likely to develop an endocrine disorder compared with siblings even when not treated with radiation (Mostoufi-Moab et al., 2016). Within the general population, memory and attention problems are common features of patients who suffer from inadequate hypothalamic-pituitary-adrenal (HPA) axis function (Schultebraucks et al., 2015). Dehydroepiandrosterone-sulfate (DHEAS) is one of the most abundant steroid hormones produced by the adrenal cortex; it is an androgen precursor that is almost exclusively produced by the adrenals and is commonly used as a marker of HPA function in adults (Nasrallah \& Arafah, 2003). Lower DHEAS levels have been found to predict accelerated global cognitive decline in elderly patients independent of age (Valenti et al., 2009). A blunted DHEAS response to stress has also been associated with poorer cognition, mood and sleep quality (Jackowska et al., 2013; Lennartsson et al., 2012). Even though there is evidence to support the presence of adrenal insufficiency in cancer survivors (Chemaitilly \& Sklar, 2010), there is currently no study in the literature that focuses on the association of DHEAS levels with neurocognitive function.

In this study, we report the concurrent association between DHEAS and attention problems in long-term survivors of childhood ALL. We hypothesize that in adolescent and young adult long-term survivors of ALL, lower levels of DHEAS, which may be indicative of a partial but persistent impairment of adrenal function secondary to the cancer and/or its treatment, would be associated with poorer attention performance. 


\section{Methods}

\subsection{Participants}

Long-term survivors treated on an institutional chemotherapy-only protocol (Pui et al., 2009), who were at least five years from diagnosis and over eight years of age, were eligible for this study. Survivors were excluded if they received cranial radiotherapy (CRT) for the treatment of central nervous system relapse or bone marrow transplantation, developed any relapse or a secondary cancer that required additional chemotherapy, had a pre-existing noncancer-related neurodevelopmental or genetic disorder associated with cognitive impairment, had a subsequent brain injury unrelated to their cancer, or were not proficient in English. Of 85 survivors eligible for this study, 69 survivors $(81 \%$; 35 male and 34 female survivors) agreed to participate in the study and provided serum samples. This study was approved by the institutional review board at St. Jude Children's Research Hospital, and informed consent and assent were obtained as appropriately from the parents/guardians and/or patients.

\subsection{Attention outcomes}

Survivors completed the Conners' Continuous Performance Test - $2^{\text {nd }}$ Ed. (CPT-II), a computerized sustained attention measure that yields scores for detectability, omissions, commissions, variability, perseverations, hit reaction time, vigilance and risk taking (Conners, 2001). Tests were administered by a master's-level psychological examiner, under the supervision of a board-certified licensed neuropsychologist.

\subsection{Biomarker assay}

A total of $5 \mathrm{ml}$ of blood was drawn from all participants according to standard procedures within 24 hours of attention testing. Samples were processed and the serum was removed, aliquoted and stored at -80 degrees Celsius until assay. The serum was assayed in duplicate using standardized immunoassays for DHEAS.

\subsection{Statistical analysis}

Attention measures were transformed into age-adjusted Z-scores using national normative data and presented for descriptive purposes. As there is evidence in the literature that highlights a sex effect in the relationship between sex hormones and health outcomes (Goldman \& Glei, 2007; Jackowska et al., 2013), all analyses were conducted separately in male and female survivors. Based on existing literature, we did not expect DHEAS to fall below clinical ranges in the majority of these survivors, though we did expect there to be a threshold effect between DHEAS and health outcomes. DHEAS levels were rank-ordered into tertiles by sex and the CPT raw scores were compared between survivors falling in the bottom tertile vs other tertiles using general linear modeling, adjusting for Tanner Stage (pre-pubescent Stage I vs pubescent Stages II to IV vs post-pubescent Stage IV), age at attention evaluation and time since diagnosis. A higher raw score is indicative of greater attention problems. This approach has been adopted by other studies in the literature that looked at the effect of physiological biomarkers on health outcomes (Cheung et al., 2016; Goldman \& Glei, 2007). 


\section{Results}

Survivors' demographic and clinical characteristics, as well as DHEAS values for each reference group, are presented in Table 1. As in the general population, male survivors had overall higher DHEAS levels than female survivors. A majority of the survivors had DHEAS levels that were within the normal reference range, though $8 / 35$ (22\%) male and 4/34 (12\%) female survivors had DHEAS levels that were below age-, sex- and Tanner Stage-based clinical reference for healthy children and adolescents (Elmlinger et al., 2002; Guran et al., 2015). Age was positively correlated with DHEAS level in both male ( $\mathrm{rsp}=0.81, P<0.0001$ ) and female ( $\mathrm{rsp}=0.61, P<0.0001)$ survivors. Body mass index was positively correlated with DHEAS in male ( $\mathrm{rsp}=0.67, P<0.0001$ ), but not in female survivors ( $\mathrm{rsp}=0.22, P=0.20$ ).

Tanner Stage (Stage I vs Stage II to IV vs Stage V) is associated with DHEAS levels in both male (median[IQR] ug/dL 25.2 [7.9 - 69.1] vs 101.8 [69.2 - 123.8] vs 144.1 [127.5 245.1]; $P<0.0001)$ and female (39.9 [18.3 - 52.4] vs 82.7 [64.2 - 139.9] vs 108.0 [83.9 153.1]; $P=0.003$ ) survivors.

Survivors scored below normative reference on multiple measures of attention (Table 1). After adjusting for Tanner Stage, age at evaluation and time since diagnosis, male survivors in the bottom tertile of DHEAS level demonstrated worse attention performance than those in other tertiles (omissions mean Z-score -1.24 vs. $-0.62, P=0.03$; variability -1.38 vs. $-0.50, P=0.008$; perseverations -1.47 vs. $-0.67, P=0.01$; vigilance -1.38 vs. $-0.32, P=0.04$; Figure 1). Association between DHEAS and attention measures were not found in female survivors.

\section{Discussion}

To our knowledge this is one of the first studies to explore the potential contribution of HPA axis function to neurocognitive outcomes among cancer survivors. Sex differences were observed in the association between DHEAS and neurocognitive function. Male survivors with low-normal DHEAS levels demonstrated worse attention than male survivors with higher DHEAS.

The majority of the survivors had DHEAS levels that were within the normal range. This population of ALL survivors was not expected to have major adrenal complications as they were not treated with CRT or neurosurgery, which are significant predictors of HPA dysfunction in cancer survivors (Chemaitilly \& Sklar, 2010). However, survivors within the bottom DHEAS tertile demonstrated low-normal DHEAS levels based on their age groups, which may imply some partial form of persistent adrenal insufficiency that may be induced by alkylating agents and glucocorticoids (Chemaitilly \& Sklar, 2010). Our preliminary results suggests that in survivors of childhood ALL who are already at-risk for cancer treatment-related neurotoxicity, subclinical disruption in the HPA axis during long-term cancer survivorship may be associated with neurocognitive deficits. These neurocognitive symptoms may be evident before clinical effects of adrenal insufficiency surface. This finding warrants validation in larger prospective studies. 
Male survivors in the bottom DHEAS tertile performed worse than those in other tertiles on attention measures. Emerging literature highlights DHEAS as a neuroactive, neuromodulating and neuroprotective steroid. Both animal and human studies in aging populations have demonstrated that the biological activities of DHEAS include promoting neurite growth, neuroneogenesis and neural survival (Maninger et al., 2009; Valenti et al., 2009). It has even been proposed that DHEAS offers protection against apoptosis and exerts antagonistic effects on oxidants (Jacob et al., 2010). There is now growing evidence that points to inflammation and oxidative stress as one of the pathophysiological processes behind cancer-related late effects (Cole et al., 2015). The potential anti-oxidative effect of DHEAS may explain why low levels are associated with poorer neurocognitive outcomes in survivors.

The observed association between lower levels of DHEAS and poorer attention performance in male survivors only is not unexpected. Because male survivors have higher physiologic DHEAS levels in comparison with female survivors, they may be more dependent upon DHEAS function, and we speculate that they may be more vulnerable to partial adrenal deficiencies than female survivors (Guran et al., 2015). Moreover, male survivors at the bottom DHEAS tertile had much worse attention scores than female survivors from the bottom tertile, thus suggesting that male survivors may be more sensitive to the effects of low DHEAS levels. The effect of sex on associations between DHEAS and neurological outcomes has been inconsistent. One study found that the physiological and psychological benefits of DHEAS secretion during acute stress did not differ between sexes (Lennartsson et al., 2012), while another reported associations between DHEAS and declines in various dimensions of psychological and physical health in men but not in women (Goldman \& Glei, 2007). Certain domains of neurocognitive function also differ by sex in survivors of childhood cancer treated with CRT, though inconsistent findings are observed among survivors treated with chemotherapy only (Armstrong et al., 2007). Understanding sex differences in how DHEAS affects the developing brain may help to identify underlying pathophysiology behind these late effects in survivors.

Several limitations should be considered in the interpretation of these results. DHEAS as a single marker may not be sufficient to confirm adrenal insufficiency in survivors, even though it is arguably one of the best-known hormonal predictors of health outcomes in children and adolescents, with well-defined age- and sex-related reference ranges. Like any other stress hormones, DHEAS is subjected to daily fluctuations. However, as compared to cortisol and DHEA, DHEAS is cleared less rapidly from the bloodstream, has less diurnal variation and has a wide clinical range across different age groups. We do not expect circadian-related changes to be large enough to account for large variations in survivors' neurocognitive performance in this study.

Our results, albeit preliminary, support the known effects of adrenal insufficiency on neurocognition in the general population, and may suggest increased vulnerability to partial insufficiency in cancer survivors. Our finding highlights the need for future research to explore the role of adrenal function in cancer-related symptoms. We propose that the current results should be validated in larger prospective studies. Future studies should also evaluate 
whether the effect of sleep on neurocognitive function may be mediated by HPA axis reactivity to stress and reduced DHEAS production.

\title{
Acknowledgments
}

Funding:

This study was supported by the National Institute of Mental Health (Grant No. MH085849 to K.R.K.), the National Cancer Institute (Grant No. CA195547 to M.M.H. and L.L.R.), and by the American Lebanese Syrian Associated Charities.

\section{Abbreviations}

\author{
CPT Conners' Continuous Performance Test \\ DHEAS Dehydroepiandrosterone-sulfate
}

\section{References}

Armstrong GT, Sklar CA, Hudson MM, Robison LL. Long-term health status among survivors of childhood cancer: Does sex matter? J. Clin. Oncol. 2007; 25:4477-4489. [PubMed: 17906209]

Chemaitilly W, Sklar CA. Endocrine complications in long-term survivors of childhood cancers. Endocr. Relat. Cancer. 2010; 17:R141-R159. [PubMed: 20453080]

Cheung YT, Edelmann MN, Mulrooney DA, Green DM, Chemaitilly W, John N, et al. Uric Acid and Neurocognitive Function in Survivors of Childhood Acute Lymphoblastic Leukemia Treated with Chemotherapy Only. Cancer Epidemiol Biomarkers Prev. 2016; 25:1259-1267. [PubMed: 27345588]

Cole PD, Finkelstein Y, Stevenson KE, Blonquist TM, Vijayanathan V, Silverman LB, et al. Polymorphisms in genes related to oxidative stress are associated with inferior cognitive function after therapy for childhood acute lymphoblastic leukemia. J. Clin. Oncol. 2015; 33:2205-2211. [PubMed: 25987702]

Conners, CK. Conners' Continuous Performance Performance Test II. Multi-Health Systems; North Tonawanda, NY: 2001.

Elmlinger MW, Kuhnel W, Ranke MB. Reference ranges for serum concentrations of lutropin (LH), follitropin (FSH), estradiol (E2), prolactin, progesterone, sex hormone-binding globulin (SHBG), dehydroepiandrosterone sulfate (DHEAS), cortisol and ferritin in neonates, children and young adults. Clin Chem Lab Med. 2002; 40:1151-1160. [PubMed: 12521235]

Goldman N, Glei DA. Sex differences in the relationship between DHEAS and health. Exp. Gerontol. 2007; 42:979-987. [PubMed: 17604586]

Guran T, Firat I, Yildiz F, Kaplan Bulut I, Dogru M, Bereket A. Reference values for serum dehydroepiandrosterone-sulphate in healthy children and adolescents with emphasis on the age of adrenarche and pubarche. Clin. Endocrinol (Oxf). 2015; 82:712-718. [PubMed: 25208296]

Jackowska M, Kumari M, Steptoe A. Sleep and biomarkers in the english longitudinal study of ageing: Associations with C-reactive protein, fibrinogen, dehydroepiandrosterone sulfate and hemoglobin. Psychoneuroendocrinology. 2013; 38:1484-1493. [PubMed: 23352806]

Jacob MHVM, Janner DDR, Jahn MP, Kucharski LC, Belló-Klein A, Ribeiro MFM. Age-related effects of DHEA on peripheral markers of oxidative stress. Cell. Biochem. Funct. 2010; 28:52-57. [PubMed: 19924683]

Krull KR, Cheung YT, Fellah S, Liu W, Ogg RJ, Srivastava D, et al. Chemotherapy Pharmacodynamics, Neuroimaging and Neurocognitive Outcomes in Long-Term Survivors of Childhood Acute Lymphoblastic Leukemia. J. Clin. Oncol. 2016; 34:2644-2653. [PubMed: 27269941] 
Lennartsson AK, Kushnir MM, Bergquist J, Jonsdottir IH. DHEA and DHEA-S response to acute psychosocial stress in healthy men and women. Biol. Psychol. 2012; 90:143-149. [PubMed: 22445967]

Maninger N, Wolkowitz OM, Reus VI, Epel ES, Mellon SH. Neurobiological and neuropsychiatric effects of dehydroepiandrosterone (DHEA) and DHEA sulfate (DHEAS). Front. Neuroendocrinol. 2009; 30:65-91. [PubMed: 19063914]

Mostoufi-Moab S, Seidel K, Leisenring WM, Armstrong GT, Oeffinger KC, Stovall M, et al. Endocrine Abnormalities in Aging Survivors of Childhood Cancer: A Report From the Childhood Cancer Survivor Study. J Clin Oncol. 2016; 34:3240-3247. [PubMed: 27382091]

Nasrallah MP, Arafah BM. The Value of Dehydroepiandrosterone Sulfate Measurements in the Assessment of Adrenal Function. J. Clin. Endocrinol Metab. 2003; 88:5293-5298. [PubMed: 14602764]

Pui CH, Campana D, Pei D, Bowman WP, Sandlund JT, Kaste SC, et al. Treating childhood acute lymphoblastic leukemia without cranial irradiation. N. Engl. J. Med. 2009; 360:2730-2741. [PubMed: 19553647]

Schultebraucks K, Wingenfeld K, Heimes J, Quinkler M, Otte C. Cognitive function in patients with primary adrenal insufficiency (Addison's disease). Psychoneuroendocrinology. 2015; 55:1-7. [PubMed: 25705797]

Valenti G, Ferrucci L, Lauretani F, Ceresini G, Bandinelli S, Luci M, et al. Dehydroepiandrosterone sulfate and cognitive function in the elderly: The InCHIANTI study. J. Endocrinol. Invest. 2009; 32:766-772. [PubMed: 19620821] 


\section{Highlights}

- Childhood acute lymphoblastic leukemia (ALL) survivors have endocrine complications

- Male survivors with low dehydroepiandrosterone-sulfate (DHEAS) had worse attention

- Partial adrenal insufficiency may impact neurocognitive function in ALL survivors

- Males may be more vulnerable to neurocognitive effects of low DHEAS than females 

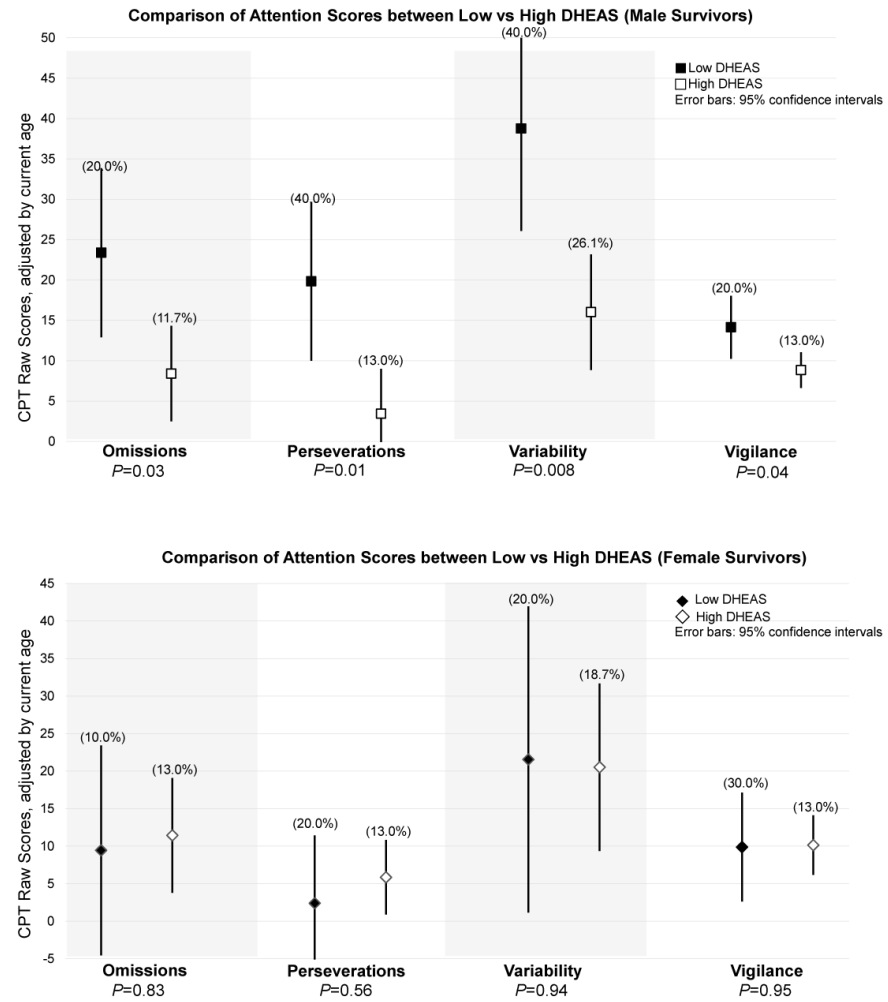

Figure 1. Association between Attention Scores and DHEAS

Caption: Attention performance is represented by the CPT raw scores; a higher score is indicative of worse attention performance. $P$ denotes the statistical significance for comparing attention performance between survivors in the bottom tertile of DHEAS and survivors in other tertiles, adjusted for Tanner Stage, age at evaluation and time since diagnosis, using general linear modelling. Error bars represent the $95 \%$ confidence interval of the adjusted raw scores. Associations are tested in male and female survivors separately. Results show that male survivors in the bottom tertile of DHEAS level demonstrated worse attention performance than those in other tertiles. Association between DHEAS and attention measures were not found in female survivors.

Rates of impairment (\%) are presented above the error bars for each group. Impairment is defined as those with age-adjusted Z-scores falling below the bottom $10^{\text {th }}$ percentile of the population norms. 
Table 1

Survivor Demographics and Treatment Characteristics

\begin{tabular}{|c|c|c|c|c|c|}
\hline & & \multicolumn{2}{|c|}{ Male survivors (N=35) } & \multicolumn{2}{|c|}{ Female survivors $(\mathrm{N}=34)$} \\
\hline & & $\mathbf{N}(\%)$ & $\begin{array}{l}\text { Mean (SD) } \\
\text { [Range] }\end{array}$ & $\mathbf{N}(\%)$ & $\begin{array}{l}\text { Mean (SD) } \\
\text { [Range] }\end{array}$ \\
\hline \multicolumn{6}{|l|}{ Demographics/Clinical } \\
\hline \multirow[t]{3}{*}{ Race } & White & $30(86)$ & & $26(76)$ & \\
\hline & Black & $5(14)$ & & $6(18)$ & \\
\hline & Others & $0(0)$ & & $2(6)$ & \\
\hline Ethnicity & Hispanic & $2(6)$ & & $4(12)$ & \\
\hline \multirow[t]{3}{*}{ Tanner Stages } & Stage I (Pre-pubescent) & $12(34)$ & & $12(35)$ & \\
\hline & Stage II to IV (Pubescent) & $8(23)$ & & $10(30)$ & \\
\hline & Stage V (Post-pubescent) & $15(43)$ & & $12(35)$ & \\
\hline Current age & Years & & $\begin{array}{l}14.8(5.2) \\
{[8.2-25.5]} \\
\end{array}$ & & $\begin{array}{l}14.1(4.3) \\
{[8.9-25.4]} \\
\end{array}$ \\
\hline Education & Years & & $\begin{array}{l}7.6(3.9) \\
{[2.0-14.0]}\end{array}$ & & $\begin{array}{l}7.6(3.5) \\
{[3.0-14.0]}\end{array}$ \\
\hline Body mass index & $\mathrm{kg} / \mathrm{m}^{2}$ & & $\begin{array}{l}23.1(8.3) \\
{[15.4-46.4]}\end{array}$ & & $\begin{array}{l}22.3(5.1) \\
{[14.1-36.7]}\end{array}$ \\
\hline Age at diagnosis & Years & & $\begin{array}{l}7.0(4.8) \\
{[1.2-16.5]}\end{array}$ & & $\begin{array}{l}7.0(4.5) \\
{[2.1-17.7]}\end{array}$ \\
\hline Time since diagnosis & Years & & $\begin{array}{l}7.8(2.0) \\
{[5.1-12.5]} \\
\end{array}$ & & $\begin{array}{l}7.2(1.7) \\
{[5.1-11.6]}\end{array}$ \\
\hline \multicolumn{6}{|c|}{ Treatment characteristics } \\
\hline Oral dexamethasone & $\left(\mathrm{mg} / \mathrm{m}^{2}\right)$ & & $\begin{array}{l}1147.6(326.2) \\
{[412.3-1690.1]} \\
\end{array}$ & & $\begin{array}{l}1049.1(194.9) \\
{[444.2-1534.8]} \\
\end{array}$ \\
\hline \multirow[t]{4}{*}{ Methotrexate } & IV standard-dose $\left(\mathrm{g} / \mathrm{m}^{2}\right)$ & & $\begin{array}{l}5.2(4.4) \\
{[2.7-9.4]}\end{array}$ & & $\begin{array}{l}3.3(0.8) \\
{[1.7-5.1]}\end{array}$ \\
\hline & IV high-dose (low risk arm) $\left(\mathrm{g} / \mathrm{m}^{2}\right)$ & $15(42)$ & $\begin{array}{l}11.5(3.2) \\
{[2.5-17.1]} \\
\end{array}$ & $26(76)$ & $\begin{array}{l}11.6(1.8) \\
{[9.3-16.4]} \\
\end{array}$ \\
\hline & IV high-dose (standard risk arm) $\left(\mathrm{g} / \mathrm{m}^{2}\right)$ & $20(58)$ & $\begin{array}{l}19.5(4.4) \\
{[7.4-26.3]}\end{array}$ & $8(24)$ & $\begin{array}{l}20.8(5.2) \\
{[15.6-29.3]}\end{array}$ \\
\hline & IT (ml) & & $\begin{array}{l}180.1(52.5) \\
{[60.0-288.0]}\end{array}$ & & $\begin{array}{l}148.6(35.2) \\
{[93.0-276.0]}\end{array}$ \\
\hline IT MHA chemotherapy & Number of counts & & $\begin{array}{l}15.6(4.3) \\
{[11.0-24.0]}\end{array}$ & & $\begin{array}{l}13.0(3.2) \\
{[9.0-23.0]}\end{array}$ \\
\hline \multicolumn{6}{|l|}{ Attention Outcomes } \\
\hline & & $P^{*}$ & $\begin{array}{l}\text { Mean (SD) Z-scores } \\
{[\% \text { impairment] }}\end{array}$ & & $\begin{array}{l}\text { Mean (SD) Z-scores } \\
{[\% \text { impairment }]}\end{array}$ \\
\hline \multirow[t]{4}{*}{ Attention } & Detectability & 0.16 & $\begin{array}{l}-0.34(1.6) \\
{[15 \%]}\end{array}$ & & $\begin{array}{l}-0.17(0.9) \\
{[12 \%]}\end{array}$ \\
\hline & Omissions & 0.03 & $\begin{array}{l}-0.81(1.9) \\
{[24 \%]}\end{array}$ & & $\begin{array}{l}-0.18(1.2) \\
{[12 \%]}\end{array}$ \\
\hline & Commissions & 0.09 & $\begin{array}{l}-0.49(1.5) \\
{[12 \%]}\end{array}$ & & $\begin{array}{l}-0.18(1.2) \\
{[15 \%]}\end{array}$ \\
\hline & Variability & 0.02 & $\begin{array}{l}-0.77(1.6) \\
{[30 \%]}\end{array}$ & & $\begin{array}{l}-0.09(1.1) \\
{[12 \%]}\end{array}$ \\
\hline
\end{tabular}




\begin{tabular}{|c|c|c|c|c|c|}
\hline & & \multicolumn{2}{|c|}{ Male survivors $(\mathbf{N}=35)$} & \multicolumn{2}{|c|}{ Female survivors $(\mathrm{N}=34)$} \\
\hline & & $\mathbf{N}(\%)$ & $\begin{array}{l}\text { Mean (SD) } \\
\text { [Range] }\end{array}$ & $\mathbf{N}(\%)$ & $\begin{array}{l}\text { Mean (SD) } \\
\text { [Range] }\end{array}$ \\
\hline & Perseverations & 0.05 & $\begin{array}{l}-0.91(2.5) \\
{[21 \%]}\end{array}$ & & $\begin{array}{l}-0.18(1.1) \\
{[15 \%]}\end{array}$ \\
\hline & Hit reaction time & 0.35 & $\begin{array}{l}-0.27(1.5) \\
{[12 \%]}\end{array}$ & & $\begin{array}{l}0.08(0.8) \\
{[9 \%]}\end{array}$ \\
\hline & Vigilance & 0.05 & $\begin{array}{l}-0.65(1.5) \\
{[21 \%]}\end{array}$ & & $\begin{array}{l}-0.04(1.2) \\
{[18 \%]}\end{array}$ \\
\hline & Risk taking & 0.86 & $\begin{array}{l}-0.19(1.4) \\
{[6 \%]}\end{array}$ & & $\begin{array}{l}0.20(0.4) \\
{[4 \%]}\end{array}$ \\
\hline \multicolumn{6}{|l|}{ DHEAS levels } \\
\hline & & $\mathbf{N}$ & Median (IQR) ug/dL & $\mathbf{N}$ & Median (IQR) ug/dL \\
\hline Overall & & 35 & $102.75(47.0-144.1)$ & 34 & $72.77(47.3-120.6)$ \\
\hline Bottom tertile & & 11 & $23.54(6.4-47.0)$ & 11 & $31.42(16.3-47.3)$ \\
\hline Other tertile & & 24 & $130.25(101.8-215.5)$ & 23 & $108.18(69.5-142.2)$ \\
\hline
\end{tabular}

Abbreviations: DHEAS, Dehydroepiandrosterone-sulfate; HD, High-dose; IT MHA, intrathecal injection of methotrexate plus hydrocortisone plus cytarabine; IV, intravenous; SD, standard deviation; IQR, intra-quartile range.

\# With the exception of IT chemotherapy, all drugs are presented in cumulative doses. HD IV methotrexate was calculated separately from standard dose. HD IV methotrexate was defined as daily dose of more than $1 \mathrm{~g} / \mathrm{m}^{2}$ of IV methotrexate. $1 \mathrm{~mL}$ of IT injection contains $1 \mathrm{mg}$ of methotrexate, $2 \mathrm{mg}$ of hydrocortisone and $3 \mathrm{mg}$ of cytarabine

Pdenotes statistical significance for comparing the age-adjusted Z-scores of overall group average to the expected population value $(\mu=0, \sigma=1.0)$ for each specific attention measure, adjusted for false discovery rate.

Group means and standard deviations are represented in age-adjusted Z scores referenced to nationally representative norms. A lower Z-score is indicative of worse functioning. Impairment is defined as a score falling below the $10^{\text {th }}$ percentile of the age-adjusted $\mathrm{Z}$ score. 